\title{
Association between Sedentary Time and Cardiovascular Risk Factors in Korean Adults
}

\author{
Jae-Hong Park', Hee-Kyung Joh ${ }^{1,2,3, *}$, Gyeong-Sil Lee', Sang-Jun Je', Soo-Hwan Cho', Soo-Jin Kim', \\ Seung-Won $\mathrm{Oh}^{4}$, Hyuk-Tae Kwon ${ }^{1}$ \\ 'Department of Family Medicine, Seoul National University Hospital, Seoul, Korea \\ ${ }^{2}$ Department of Medicine, Seoul National University College of Medicine, Seoul, Korea \\ ${ }^{3}$ Department of Family Medicine, Seoul National University Health Service Center, Seoul, Korea \\ ${ }^{4}$ Department of Family Medicine, Seoul National University Hospital Healthcare System Gangnam Center, Seoul, Korea
}

\begin{abstract}
Background: Sedentary behavior has been shown to have deleterious effects on cardiovascular outcomes. This study aimed to examine the association between sedentary time and cardiovascular risk factors in Korean adults. Methods: A cross-sectional study was performed using data from adults aged 19 years and above in the 2013 Korean National Health and Nutrition Examination Survey. Sedentary time was self-reported and categorized into quintiles. Cardiovascular risk factors, including blood pressure (BP), total cholesterol, high-density lipoprotein (HDL) cholesterol, triglycerides, low-density lipoprotein cholesterol, and fasting glucose levels, were categorized into dichotomous variables according to the Adult Treatment Panel III criteria. Multivariate logistic regression was performed with adjustment for various demographic and lifestyle variables, body mass index (BMI), waist circumference (WC), and moderate-to-vigorous physical activity. Complex sampling design was used, and survey weights and sample design variables were applied in analyses.

Results: A total of 3,301 individuals were included in the analyses, and mean sedentary time was $6.1 \mathrm{~h} / \mathrm{d}$. Prolonged sedentary time was significantly associated with high diastolic BP (top vs. bottom quintile: adjusted odds ratio [OR], $1.71 ; 95 \%$ confidence interval [CI], 1.09-2.68; $\mathrm{P}_{\text {trend }}=0.03$ ) and low HDL cholesterol level (top vs. bottom quintile: adjusted OR, 1.42; 95\% CI, 1.02-1.98; $\mathrm{P}_{\text {trend }}=0.02$ ) after adjustment for BMI, WC, moderate-to-vigorous physical activity, and other variables. No significant associations were found between sedentary time and other cardiovascular risk factors.

Conclusion: Prolonged sedentary time was significantly associated with high diastolic BP and low HDL cholesterol level in Korean adults. The associations were independent of general and abdominal obesity and moderate-to-vigorous physical activities.
\end{abstract}

Keywords: Sedentary Lifestyle; Exercise; Cardiovascular Diseases; Risk Factors

Received: April 8, 2016, Revised: October 21, 2016, Accepted: October 25, 2016

*Corresponding Author: Hee-Kyung Joh https://orcid.org/0000-0003-3854-7012

Tel: +82-2-880-5350, Fax: +82-2-880-9274, E-mail: hkjoh@snu.ac.kr 


\section{INTRODUCTION}

Sedentary lifestyles have become common over the past few decades with the modernization of the society. ${ }^{1)}$ Sedentary behavior, which is distinct from physical inactivity, is defined as a failure to meet the current physical activity recommendations. ${ }^{2}$ Individuals who meet the current physical activity recommendations may also have negative health outcomes if they spend excessive time sitting. The Korea Ministry of Health and Welfare recommends less than $2 \mathrm{~h} / \mathrm{d}$ of sitting time and performing slight physical activities. At least 10 minutes per one bout of moderate-to-vigorous physical activity (MVPA) is also recommended. ${ }^{3)}$

Numerous studies suggest that high amounts of sedentary behavior are associated with increased risk of morbidity and mortality regardless of MVPA level. ${ }^{2)}$ Increasing evidences show that sedentary behavior is an independent risk factor of cardiovascular disease regardless of MVPA. ${ }^{4)}$ A previous study found linear associations between sedentary time and low high-density lipoprotein (HDL) cholesterol, as well as high triglycerides, waist circumference, C-reactive protein, and insulin. ${ }^{5)}$ Similarly, consistent positive associations between all sedentary behaviors and triglycerides, low-density lipoprotein (LDL) cholesterol, or total cholesterol were observed in normal weight individuals. ${ }^{6)}$ In contrast, a recent study has suggested that cardiovascular disease risk is non-linearly associated with sedentary time with an increased risk only at extremely high levels $(>10 \mathrm{~h} / \mathrm{d}))^{7)}$ Another study found no associations between cardiovascular biomarkers and sedentary time. ${ }^{8)} \mathrm{A}$ study among Korean children and adolescents, aged 12 to 18 years, found that watching TV is significantly associated with risks of overweight, high abdominal adiposity, and low HDL cholesterol; an additional hour spent playing video games also increases the risk of high abdominal adiposity. ${ }^{9)}$

Few studies about the associations between sedentary time and cardiovascular risks among Korean adults have been conducted. Therefore, we examined the associations between sedentary time and cardiovascular risk factors in a large representative sample of Korean adults in this study.

\section{METHODS}

\section{Participants}

Data were collected from the 2013 Korean National Health and Nutrition Examination Survey (KNHANES) VI. The KNHANES is a nationwide cross-sectional survey designed to gather information on the sociodemographic status, health examination, and health behaviors, including physical activities, among representative samples of Koreans. To sample representative individuals of the civilian, non-institutionalized Korean population, we used a complex multistage probability sampling design. Among the 8,018 individuals participated in 2013 KNHANES, we included 6,273 participants aged 19 years and above. Patients who had no information on sedentary time (n=934) and pregnant women $(n=31)$ were excluded from the analyses. Those who were diagnosed with hypertension, diabetes, dyslipidemia, stroke, myocardial infarction, angina, osteoarthritis, rheumatoid arthritis, and any cancers $(n=2,007)$ were also excluded to reduce the possibility of reverse-causality. Data from 3,301 participants were available for the present analysis.

\section{Measurements}

\section{1) Cardiovascular risk factors}

Biomarkers of cardiovascular risk included systolic blood pressure (SBP), diastolic blood pressure (DBP), total cholesterol, HDL cholesterol, LDL cholesterol, triglycerides, and fasting glucose. Blood pressure was measured 3 times after at least 5 minutes of rest in a sitting position with a mercury sphygmomanometer by a trained staff. Mean SBP and DBP were calculated as the mean of the last 2 measurements. Total cholesterol and triglycerides were measured with the enzymatic method. HDL cholesterol and LDL cholesterol levels were measured using the homogeneous enzymatic colorimetric method. Fasting glucose level was measured with the hexokinase method. All laboratory tests were performed with COBAS 8000 C702 (Roche, Mannheim, Germany). Measurements with 8 or more fasting hours (12 hours for triglycerides) were used for analyses. Direct LDL cholesterol level was measured when triglyceride levels were higher than $200 \mathrm{mg} / \mathrm{dL}$, and calculated LDL cholesterol level was obtained using the Friedewald equation only when triglyceride levels were $<200 \mathrm{mg} / \mathrm{dL}$. In this study, we performed either direct or calculated measurements. Measurements of cardiovascular biomarkers were categorized into dichotomous variables according to the Adult Treatment Panel (ATP) III criteria. High SBP was defined as $130 \mathrm{~mm} \mathrm{Hg}$ or higher, and high DBP was defined as $85 \mathrm{~mm} \mathrm{Hg}$ or higher. High SBP or DBP is considered high blood pressure. High total cholesterol level was defined as $200 \mathrm{mg} / \mathrm{dL}$ or higher. A cholesterol level of less than $40 \mathrm{mg} / \mathrm{dL}$ in men or less than $50 \mathrm{mg} / \mathrm{dL}$ in women indicates a low HDL cholesterol. High triglyceride and high LDL cholesterol levels were defined as $150 \mathrm{mg} / \mathrm{dL}$ or higher and as $130 \mathrm{mg} / \mathrm{dL}$ or higher, respectively. A glucose level of $100 \mathrm{mg} / \mathrm{dL}$ or higher is considered high fasting glucose level.

\section{2) Sedentary time and physical activity}

Sedentary time and physical activities (vigorous, moderate, and walking) were assessed by self-reported questionnaire based on the International Physical Activity Questionnaire (IPAQ). Participants were asked the question: "How much time do you usually spend sitting on a typical day for the last seven days ( $\mathrm{min} / \mathrm{d}$ )?" Total sedentary time per day was used as both continuous and categorical (quintile) variables. Quintile cutoff points for the total sedentary time were 3.0, 4.5, 6.0, and $9.5 \mathrm{~h} / \mathrm{d}$. MVPA (metabolic equivalent of task [MET]-min/wk) was calculated using the guideline for data processing and analysis of the IPAQ short form ${ }^{10)}$ and was then categorized into tertiles.

\section{3) Other covariates}

The following sociodemographic and lifestyle covariates were consid- 
ered as possible confounders: age, sex, education level (below high school/high school/above high school), income level (quartiles), marital status (married/single/others), type of occupation (inactive/active), residence (urban/rural), menopausal status (premenopause/ menopause), smoking status (non-smoker/ex-smoker/current smoker), alcohol use (none/moderate/heavy), calorie intake (tertiles), body mass index (BMI, low to normal/overweight/obese), and waist circumference (normal/abdominal obesity).

Income levels were categorized into quartiles using monthly average household equivalent income (monthly average household income/square root of number of household members) by sex and age groups. Marital status was categorized into married, single, and others (bereaved, divorced, separated, or no response). Types of occupation were dichotomized into inactive and active jobs from seven job categories based on MVPA of each category. Inactive jobs included manager, specialist, office worker, and unemployed (housewife, student); active jobs included service worker and seller, foster and fishery worker, machine operator, assembler, and simple labor worker. Participants who had smoked less than 100 cigarettes in their entire lives were regarded as non-smoker. Alcohol use groups were classified according to the National Institute on Alcohol Abuse and Alcoholism criteria. ${ }^{11)}$ Calorie intake was categorized into tertiles, and no data group on nutrition existed. BMI was calculated by weight divided by the square of height. The cutoff points for overweight and obesity were 23 and $25 \mathrm{~kg} /$ $\mathrm{m}^{2}$, respectively, based on the criteria for Asians. Waist circumference cutoff points were $90 \mathrm{~cm}$ in men and $85 \mathrm{~cm}$ in women.

\section{4) Statistical analysis}

Weights and sample design variables were applied in analyses to take into account a complex, multistage, probability sampling design of KNHANES. Weighted descriptive characteristics (means, standard errors, proportions) were calculated using the guideline for 2013 KNHANES analysis. To compare sociodemographic variables by sex, we conducted t-tests and chi-square tests. Dependent variables (SBP, DBP, total cholesterol, HDL cholesterol, triglycerides, LDL cholesterol, and fasting glucose) were categorized into dichotomous variables according to the ATP III criteria. Self-reported sedentary time was analyzed as continuous and categorical variables (quintiles). Multivariate logistic regression was performed with adjustment for age, sex, education level, income level, marital status, type of occupation, residence, menopausal status, smoking status, alcohol use, calorie intake, BMI, waist circumference, and MVPA. All statistical analyses were performed using Stata ver. 14.1 for Windows (Stata Corp., College Station, TX, USA). $\mathrm{P}<0.05$ was considered statistically significant.

\section{RESULTS}

\section{Characteristics of Study Participants}

The mean \pm standard error age of the 3,301 participants was $39.5 \pm 0.3$ years. The mean sedentary time among participants was $6.1 \mathrm{~h} / \mathrm{d}(6.3$ $\mathrm{h} / \mathrm{d}$ in men, $6.0 \mathrm{~h} / \mathrm{d}$ in women; $\mathrm{P}<0.05$ ) (Table 1). Men reported more
Table 1. Sociodemographic characteristics of study participants (2013 Korean National Health and Nutrition Examination Survey)

\begin{tabular}{|c|c|c|c|c|}
\hline Characteristic & $\begin{array}{c}\text { Total } \\
(\mathrm{N}=3,301)\end{array}$ & $\begin{array}{c}\text { Men } \\
(\mathrm{N}=1,465)\end{array}$ & $\begin{array}{c}\text { Women } \\
(\mathrm{N}=1,836)\end{array}$ & P-value ${ }^{*}$ \\
\hline Age (y) & $39.5 \pm 0.3$ & $39.4 \pm 0.4$ & $39.5 \pm 0.4$ & 0.84 \\
\hline $19-29$ & $666(27.3)$ & $300(28.7)$ & 366 (25.8) & $<0.05$ \\
\hline 30-39 & $858(26.1)$ & $354(25.5)$ & $504(26.6)$ & \\
\hline $40-49$ & 830 (24.3) & 345 (22.6) & $485(26.1)$ & \\
\hline $50-59$ & $536(14.7)$ & $236(14.8)$ & 300 (14.6) & \\
\hline $60-69$ & $242(4.7)$ & $146(5.7)$ & $96(3.6)$ & \\
\hline$\geq 70$ & $169(3.0)$ & $84(2.7)$ & $85(3.3)$ & \\
\hline \multicolumn{5}{|l|}{ Education } \\
\hline Below high school & $521(12.0)$ & $218(10.7)$ & 303 (13.5) & $<0.001$ \\
\hline High school & $1,057(32.1)$ & $441(29.9)$ & $616(34.5)$ & \\
\hline College or above & $1,722(55.9)$ & $806(59.4)$ & $916(52.1)$ & \\
\hline \multicolumn{5}{|l|}{ Income $^{\dagger}$} \\
\hline Quartile 1 (low) & 823 (23.6) & $175(23.2)$ & $221(24.0)$ & 0.90 \\
\hline Quartile 2 & 824 (26.3) & 395 (26.7) & $474(25.9)$ & \\
\hline Quartile 3 & 820 (25.1) & $420(25.4)$ & 518 (24.8) & \\
\hline Quartile 4 (high) & $820(25.1)$ & $469(24.8)$ & $615(25.3)$ & \\
\hline \multicolumn{5}{|l|}{ Marital status } \\
\hline Married & $2,294(63.7)$ & 1,007 (61.0) & $1,287(66.5)$ & $<0.001$ \\
\hline Single & 778 (30.7) & 394 (35.3) & $384(25.7)$ & \\
\hline Others ${ }^{\ddagger}$ & $229(5.7)$ & $64(3.8)$ & $165(7.8)$ & \\
\hline \multicolumn{5}{|l|}{ Occupation ${ }^{\S}$} \\
\hline Inactive job & $2,062(61.9)$ & $761(52.9)$ & $1,301(71.5)$ & $<0.001$ \\
\hline Active job & $1,234(38.1)$ & 699 (47.1) & 535 (28.5) & \\
\hline \multicolumn{5}{|l|}{ Residence } \\
\hline Urban & $2,707(82.7)$ & 1,186 (82.0) & 1,521 (83.4) & 0.22 \\
\hline Rural & 594 (17.3) & 279 (18.0) & $315(16.6)$ & \\
\hline \multicolumn{5}{|l|}{ Menopause } \\
\hline Premenopause & - & - & 1,390 (80.4) & \\
\hline Postmenopause & - & - & 437 (19.6) & \\
\hline \multicolumn{5}{|l|}{ Smoking } \\
\hline Non-smoker & 2,056 (58.9) & $398(30.9)$ & 1,658 (89.1) & $<0.001$ \\
\hline Ex-smoker & 477 (14.2) & 407 (23.5) & $70(4.2)$ & \\
\hline Current smoker & 767 (26.9) & $660(45.7)$ & $107(6.7)$ & \\
\hline \multicolumn{5}{|l|}{ Alcohol } \\
\hline None & 726 (18.8) & $199(11.9)$ & 527 (26.3) & $<0.001$ \\
\hline Moderate & $2,045(64.2)$ & $926(65.4)$ & $1,119(63.0)$ & \\
\hline Heavy & $529(16.9)$ & $340(22.8)$ & $190(10.6)$ & \\
\hline Calorie intake (kcal/d)" & $2,199 \pm 24.8$ & $2,555 \pm 39.7$ & $1,832 \pm 25.8$ & $<0.001$ \\
\hline Tertile 1 (low) & 969 (31.4) & 216 (18.3) & $753(44.8)$ & $<0.001$ \\
\hline Tertile 2 & 968 (31.4) & $381(28.8)$ & 587 (34.1) & \\
\hline Tertile 3 (high) & 968 (37.1) & $624(52.8)$ & $344(20.9)$ & \\
\hline No data & $396(0.1)$ & $244(0.1)$ & $152(0.2)$ & \\
\hline Body mass index $\left(\mathrm{kg} / \mathrm{m}^{2}\right)$ & $23.3 \pm 0.1$ & $24.1 \pm 0.1$ & $22.5 \pm 0.1$ & $<0.001$ \\
\hline$<23.0$ & $1,679(50.6)$ & $576(40.3)$ & $1,103(61.6)$ & $<0.001$ \\
\hline 23.0-24.9 & $719(21.3)$ & $356(23.8)$ & $363(18.7)$ & \\
\hline$\geq 25.0$ & $901(28.1)$ & $532(35.9)$ & $369(19.7)$ & \\
\hline Waist circumference (cm) & $78.7 \pm 0.24$ & $82.5 \pm 0.3$ & $74.5 \pm 0.3$ & $<0.001$ \\
\hline$<90$ (men), 85 (women) & 2,769 (84.1) & $1,157(80.0)$ & $1,612(88.4)$ & $<0.001$ \\
\hline$\geq 90$ (men), 85 (women) & $528(16.0)$ & $308(20.0)$ & $220(11.6)$ & \\
\hline MVPA" (MET-min/wk) & $2,238 \pm 67.5$ & $2,714 \pm 103.7$ & $1,727 \pm 69.6$ & $<0.001$ \\
\hline Tertile 1 (low) & $1,149(32.4)$ & $401(26.1)$ & $748(39.1)$ & $<0.001$ \\
\hline Tertile 2 & $1,058(32.3)$ & $452(30.9)$ & $606(33.8)$ & \\
\hline Tertile 3 (high) & $1,090(35.3)$ & $611(43.0)$ & $479(27.0)$ & \\
\hline
\end{tabular}


Table 1. Continued

\begin{tabular}{ccccl}
\hline \multicolumn{1}{c}{ Characteristic } & $\begin{array}{c}\text { Total } \\
(\mathrm{N}=3,301)\end{array}$ & $\begin{array}{c}\text { Men } \\
(\mathrm{N}=1,465)\end{array}$ & $\begin{array}{c}\text { Women } \\
(\mathrm{N}=1,836)\end{array}$ & P-value* \\
\hline Sedentary time* $(\mathrm{h} / \mathrm{d})^{*}$ & $6.1 \pm 0.1$ & $6.3 \pm 0.1$ & $6.0 \pm 0.1$ & $<0.05$ \\
Quintile 1 (low) $(<3.0)$ & $494(15.0)$ & $234(16.2)$ & $260(13.5)$ & $<0.001$ \\
Quintile 2 (3.0-4.5) & $793(22.2)$ & $328(20.0)$ & $465(24.5)$ & \\
Quintile 3 (4.5-6.0) & $760(22.8)$ & $315(20.9)$ & $445(24.8)$ & \\
Quintile 4 (6.0-9.5) & $592(18.3)$ & $266(19.1)$ & $326(17.4)$ & \\
Quintile 5 (high) $(\geq 9.5)$ & $662(21.9)$ & $322(23.8)$ & $340(19.8)$ & \\
\hline
\end{tabular}

Values are presented as mean \pm standard error or number (\%), unless otherwise indicated after survey analysis. The sum of each subgroup is not same because of missing data. Statistically significant results are marked in bold.

MET, metabolic equivalent of task; MVPA, moderate-to-vigorous physical activity.

*T-tests for continuous variables and chi-square tests for categorical variables. ${ }^{\dagger}$ Quartiles were generated using monthly average household equivalent income in the same sex and age groups. "Bereaved, divorced, separated, or no response. ${ }^{\S}$ Based on average physical activity levels (METs). Inactive job includes manager, specialist, office worker, and unemployed (housewife, student), and active job includes service worker and seller, foster and fishery worker, machine operator and assembler, and simple labor worker. "Tertile cutoff points were 1,626 and 2,281. "MVPA includes walking and moderate and vigorous physical activities. Cutoff points for tertile were 594 and 2,034 MET-min/wk. **Quintile cutoff points were 3.0, 4.5, 6.0 , and $9.5 \mathrm{~h} / \mathrm{d}$. practice of MVPA than women $(\mathrm{P}<0.001)$. The average BMI was 24.1 $\mathrm{kg} / \mathrm{m}^{2}$ in men and $22.5 \mathrm{~kg} / \mathrm{m}^{2}$ in women $(\mathrm{P}<0.001)$. The proportion of abdominal obesity was higher in men $(20.0 \%)$ than in women $(11.6 \%)$ $(\mathrm{P}<0.001)$.

\section{Cardiovascular Risk Factors}

The mean values of SBP and DBP were higher in men than in women $(\mathrm{P}<0.001)$ (Table 2). Similarly, the proportions of high SBP, high DBP, or high blood pressure were higher in men than in women $(\mathrm{P}<0.001)$. Compared with women, men exhibited higher mean values and proportions of abnormal levels for most of the lipid profiles and fasting glucose. However, the proportion of low HDL cholesterol was higher in women than in men $(\mathrm{P}<0.001)$.

\section{Sedentary Time and Cardiovascular Risk Factors}

Sedentary time was inversely associated with SBP (top versus bottom quintile: adjusted odds ratio [aOR], 0.70; 95\% confidence interval [CI], 0.44 to 1.12 ; $\mathrm{P}_{\text {trend }}=0.01$ ) and fasting glucose (top versus bottom quin-

Table 2. Cardiovascular risk factors of study participants (2013 Korean National Health and Nutrition Examination Survey)

\begin{tabular}{|c|c|c|c|c|}
\hline Cardiovascular risk factors & Total & Men & Women & P-value* \\
\hline \multicolumn{5}{|l|}{$\mathrm{SBP}(\mathrm{mm} \mathrm{Hg})$} \\
\hline $\mathrm{N}$ & 3,293 & 1,461 & 1,832 & \\
\hline Mean \pm SE & $112.9 \pm 0.3$ & $116.5 \pm 0.4$ & $109.0 \pm 0.4$ & $<0.001$ \\
\hline$\geq 130$ & $429(11.4)$ & $236(13.9)$ & $193(8.7)$ & $<0.001$ \\
\hline \multicolumn{5}{|l|}{$\mathrm{DBP}(\mathrm{mm} \mathrm{Hg})$} \\
\hline $\mathrm{N}$ & 3,292 & 1,460 & 1,832 & \\
\hline Mean \pm SE & $74.6 \pm 0.3$ & $77.3 \pm 0.3$ & $71.6 \pm 0.3$ & $<0.001$ \\
\hline$\geq 85$ & $519(15.8)$ & $346(22.7)$ & $173(8.4)$ & $<0.001$ \\
\hline \multicolumn{5}{|l|}{ Blood pressure $(\mathrm{mm} \mathrm{Hg})$} \\
\hline $\mathrm{N}$ & 3,292 & 1,460 & 1,832 & \\
\hline $\mathrm{SBP} \geq 130$ or $\mathrm{DBP} \geq 85$ & $686(19.5)$ & $425(26.5)$ & $261(12.0)$ & $<0.001$ \\
\hline \multicolumn{5}{|l|}{ Total cholesterol (mg/dL) } \\
\hline $\mathrm{N}$ & 3,076 & 1,384 & 1,692 & \\
\hline Mean \pm SE & $184.8 \pm 0.8$ & $186.0 \pm 1.0$ & $183.5 \pm 0.9$ & 0.049 \\
\hline$\geq 200$ & $969(29.7)$ & 461 (31.2) & $508(28.0)$ & 0.10 \\
\hline \multicolumn{5}{|c|}{ High-density lipoprotein cholesterol (mg/dL) } \\
\hline $\mathrm{N}$ & 3,076 & 1,384 & 1,692 & \\
\hline Mean \pm SE & $48.8 \pm 0.2$ & $45.8 \pm 0.3$ & $52.1 \pm 0.3$ & $<0.001$ \\
\hline$<40$ (men), 50 (women) & $1,206(37.4)$ & $443(31.1)$ & $763(44.3)$ & $<0.001$ \\
\hline \multicolumn{5}{|l|}{ Triglycerides (mg/dL) } \\
\hline $\mathrm{N}$ & 2,417 & 1,043 & 1,374 & \\
\hline Mean \pm SE & $118.8 \pm 1.9$ & $139.9 \pm 3.2$ & $97.2 \pm 1.8$ & $<0.001$ \\
\hline$\geq 150$ & $554(23.1)$ & $338(31.0)$ & $216(15.1)$ & $<0.001$ \\
\hline \multicolumn{5}{|c|}{ Low-density lipoprotein cholesterol (mg/dL) } \\
\hline $\mathrm{N}$ & 2,512 & 1,112 & 1,400 & \\
\hline Mean \pm SE & $114.9 \pm 0.7$ & $116.2 \pm 1.1$ & $113.6 \pm 0.9$ & 0.06 \\
\hline$\geq 130$ & $752(28.1)$ & $364(30.8)$ & $388(25.2)$ & 0.007 \\
\hline \multicolumn{5}{|l|}{ Fasting glucose (mg/dL) } \\
\hline $\mathrm{N}$ & 3,074 & 1,384 & 1,690 & \\
\hline Mean \pm SE & $93.9 \pm 0.3$ & $95.4 \pm 0.4$ & $92.3 \pm 0.4$ & $<0.001$ \\
\hline$\geq 100$ & $639(20.5)$ & $376(25.2)$ & 263 (15.2) & $<0.001$ \\
\hline
\end{tabular}

Values are presented as number (\%), unless otherwise stated. Means, standard errors, and proportions are presented after survey analysis. Statistically significant results are marked in bold.

SBP, systolic blood pressure; SE, standard error; DBP, diastolic blood pressure.

${ }^{*}$ T-tests for continuous variables and chi-square tests for categorical variables. 


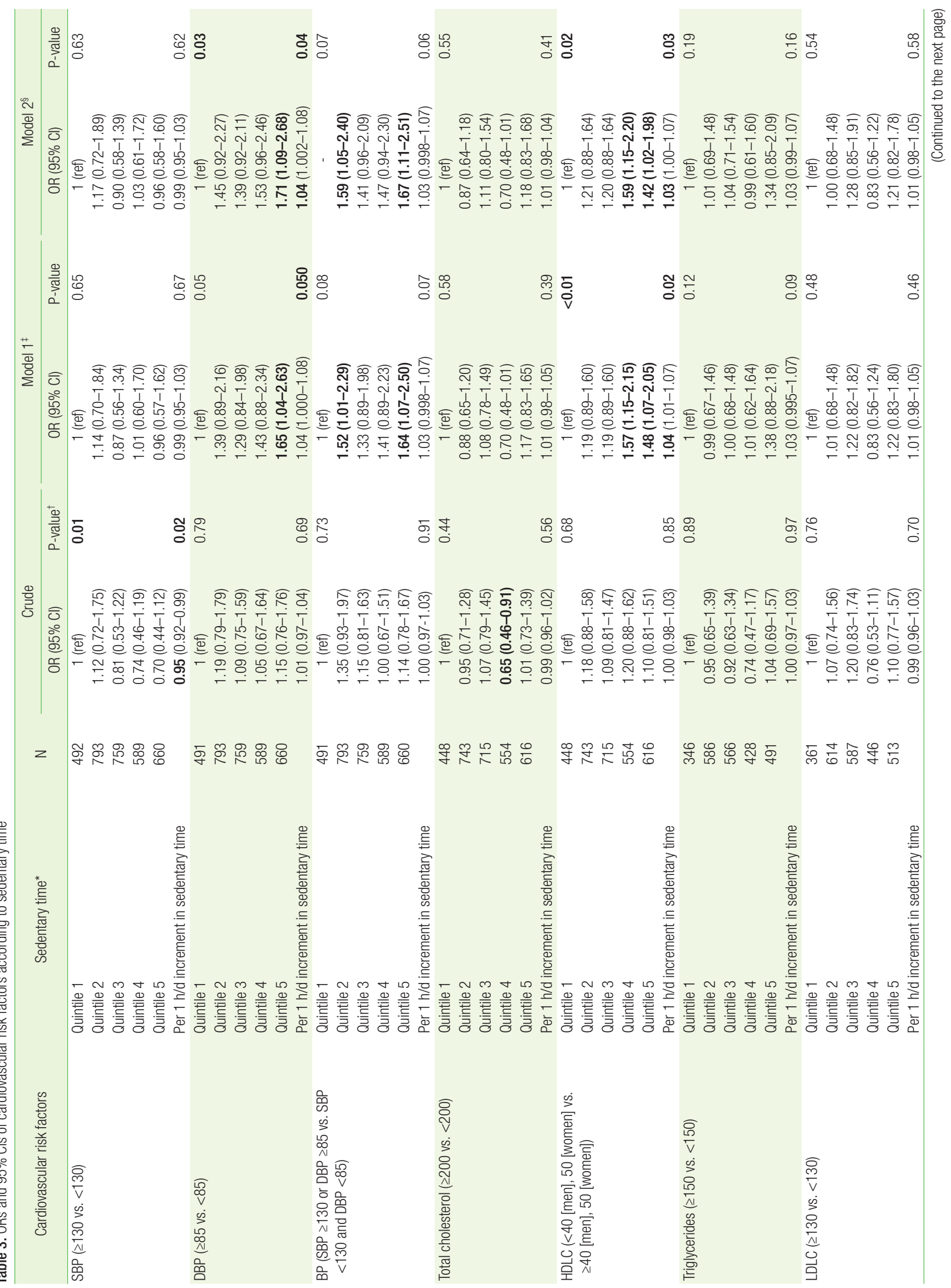




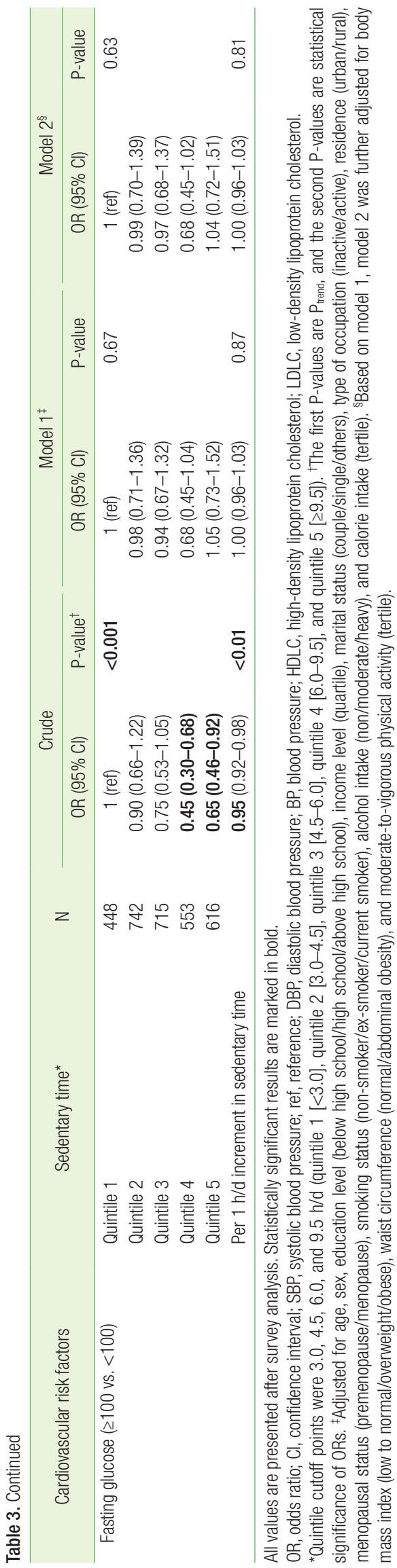

tile: aOR, $0.65 ; 95 \%$ CI, 0.46 to $0.92 ; \mathrm{P}_{\text {trend }}<0.001$ ) before adjustment (Table 3). However, after adjustment for age, sex, education level, income level, marital status, type of occupation, residence, menopausal status, smoking status, alcohol use, and calorie intake (model 1), the associations with both SBP and fasting glucose were attenuated $\left(\mathrm{P}_{\text {trend }}=0.65, \mathrm{P}_{\text {trend }}=0.67\right)$. In model 1, high DBP and low HDL cholesterol were significantly associated with prolonged sedentary time (for high DBP, top versus bottom quintile: aOR, 1.65; 95\% CI, 1.04 to 2.63; $\mathrm{P}_{\text {trend }}=0.05$; for low HDL cholesterol, top versus bottom quintile: $\mathrm{aOR}$, 1.48 ; $95 \% \mathrm{CI}, 1.07$ to $2.05 ; \mathrm{P}_{\text {trend }}<0.01$ ). These associations remained significant even after further adjustment for BMI, waist circumference, and MVPA (model 2). For high DBP, the top versus bottom quintile aOR was 1.71 (95\% CI, 1.09 to 2.68; $\mathrm{P}_{\text {trend }}=0.03$ ). For low HDL cholesterol, the top versus bottom quintile aOR was 1.42 (95\% CI, 1.02 to 1.98; $P_{\text {trend }}=0.02$ ). When sedentary time was used as a continuous variable, per 1-hour increment in sedentary time per day was significantly associated with elevated DBP and low HDL cholesterol level. The aOR of elevated DBP was 1.04 (95\% CI, 1.00 to 1.08 ; $\mathrm{P}=0.04$ ), and the aOR of low HDL cholesterol was 1.03 (95\% CI, 1.00 to 1.07; $\mathrm{P}=0.03$ ). We found no significant associations between sedentary time and other cardiovascular risk factors. When the associations were further stratified by sex, the associations between sedentary time and cardiovascular risk factors were similar in both sexes ( $\mathrm{P}_{\text {interaction }} \geq 0.05$ ) (Table 4 ).

We performed several sensitivity analyses and found almost similar results to the main analyses. When confounding variables (BMI, waist circumference, physical activity) were used as continuous variables, the associations of sedentary time with DBP $\left(P_{\text {trend }}=0.03\right)$ and HDL cholesterol $\left(\mathrm{P}_{\text {trend }}=0.01\right)$ were similar to the main results. In addition, when MVPA levels were categorized using the World Health Organization criteria ( $<600,600-3,000, \geq 3,000 \mathrm{METs})$, the associations of sedentary time with DBP $\left(\mathrm{P}_{\text {trend }}=0.03\right)$ and HDL cholesterol $\left(\mathrm{P}_{\text {trend }}=0.01\right)$ were similar to the main results.

\section{DISCUSSION}

In this cross-sectional study, prolonged sedentary time was significantly associated with high DBP and low HDL cholesterol in Korean adults. These associations were independent of general and abdominal obesity and MVPA levels.

Similar to a previous study, ${ }^{12)}$ we found that sedentary time was significantly associated with DBP, but not with SBP. The biological mechanisms that may explain the observed association between sedentary behavior and DBP remain unclear. One possibility is that sedentary behavior may affect blood pressure in different ways, and SBP can be affected by changes in peripheral vascular resistance to a much lesser extent than DBP. ${ }^{12)}$

Numerous studies ${ }^{5,6,8,13)}$ and the present study found that prolonged sedentary time is associated with low HDL cholesterol. A study among adults aged 30-50 years reported that sedentary time is associated with HDL cholesterol, but not with other lipid profiles or blood pressure. ${ }^{8)}$ Although we found no significant association with triglycerides, 
Table 4. ORs and $95 \%$ Cls of cardiovascular risk factors according to quintiles of sedentary time by sex

\begin{tabular}{|c|c|c|c|c|c|c|}
\hline \multirow{2}{*}{ Cardiovascular risk factors } & \multirow{2}{*}{ Sedentary time } & \multicolumn{2}{|c|}{ Men* } & \multicolumn{2}{|c|}{ Women $^{\dagger}$} & \multirow{2}{*}{$P$ interaction } \\
\hline & & OR $(95 \% \mathrm{Cl})$ & P for trend & $\mathrm{OR}(95 \% \mathrm{Cl})$ & $P$ for trend & \\
\hline \multirow[t]{5}{*}{ DBP ( $\geq 85$ vs. $<85$ ) } & Quintile 1 & 1 (ref) & \multirow[t]{5}{*}{0.27} & 1 (ref) & \multirow[t]{5}{*}{0.02} & \multirow[t]{5}{*}{0.09} \\
\hline & Quintile 2 & $1.40(0.90-2.18)$ & & $0.82(0.44-1.54)$ & & \\
\hline & Quintile 3 & $1.40(0.81-2.40)$ & & $1.33(0.78-2.28)$ & & \\
\hline & Quintile 4 & $1.34(0.87-2.07)$ & & $1.42(0.80-2.49)$ & & \\
\hline & Quintile 5 & $1.38(0.71-2.68)$ & & $1.98(1.08-3.63)$ & & \\
\hline \multirow{5}{*}{$\begin{array}{c}B P(S B P \geq 130 \text { or } D B P \geq 85 \text { vs. } \\
S B P<130 \text { and } D B P<85)\end{array}$} & Quintile 1 & - & \multirow[t]{5}{*}{0.47} & - & \multirow[t]{5}{*}{0.06} & \multirow[t]{5}{*}{0.09} \\
\hline & Quintile 2 & $1.46(0.97-2.19)$ & & $0.94(0.56-1.59)$ & & \\
\hline & Quintile 3 & $1.25(0.76-2.06)$ & & $1.13(0.72-1.76)$ & & \\
\hline & Quintile 4 & $1.21(0.81-1.80)$ & & $1.05(0.64-1.75)$ & & \\
\hline & Quintile 5 & $1.34(0.74-2.43)$ & & $1.89(1.05-3.40)$ & & \\
\hline \multirow{5}{*}{$\begin{array}{l}\mathrm{HDLC}(<40 \text { [men], } 50 \text { [women] vs. } \\
\geq 40 \text { [men], } 50 \text { [women]) }\end{array}$} & Quintile 1 & 1 (ref) & \multirow[t]{5}{*}{0.018} & 1 (ref) & \multirow[t]{5}{*}{0.33} & \multirow[t]{5}{*}{0.47} \\
\hline & Quintile 2 & $1.08(0.73-1.60)$ & & $1.03(0.71-1.48)$ & & \\
\hline & Quintile 3 & $1.22(0.70-2.11)$ & & $1.09(0.79-1.52)$ & & \\
\hline & Quintile 4 & $1.54(1.04-2.26)$ & & $1.27(0.86-1.89)$ & & \\
\hline & Quintile 5 & $1.55(0.90-2.66)$ & & $1.12(0.78-1.61)$ & & \\
\hline
\end{tabular}

All values are presented after survey analysis. Adjusted for age and sex, education level (below high school/high school/above high school), income (quartile), marital status (couple/single/others), occupation (inactive/active), residence (urban/rural), menopausal status (premenopause/menopause), smoking (non-smoker/ex-smoker/current smoker), alcohol (non/moderate/heavy), calorie intake (tertile), body mass index (low to normal/overweight/obese), waist circumference (normal/abdominal obesity), and moderate-tovigorous physical activity (tertile). Statistically significant results are marked in bold.

OR, odds ratio; $\mathrm{Cl}$, confidence interval; DBP, diastolic blood pressure; ref, reference; $\mathrm{BP}$, blood pressure; SBP, systolic blood pressure; HDLC, high-density lipoprotein cholesterol.

*Sedentary time quintile cutoff points were 3.0, 5.0,6.25, and $10.0 \mathrm{~h} / \mathrm{d}$ for men (quintile 1 [ $\leq 3.0$ ], quintile 2 [3.0-5.0], quintile 3 [5.0-6.25], quintile 4 [6.25-10.0], quintile 5 $[>10.0]$ ). ${ }^{~}$ Sedentary time quintile cutoff points were $3.0,4.75,6.0$, and $9.5 \mathrm{~h} / \mathrm{d}$ for women (quintile $1[\leq 3.0]$, quintile 2 [3.0-4.75], quintile 3 [4.75-6.0], quintile 4 [6.0-9.5], quintile $5[\geq 9.5])$.

some studies, including a meta-analysis, reported significant associations with triglycerides. ${ }^{5,6,13,14)}$ In the meta-analysis of studies using accelerometer, only triglycerides were associated with sedentary time among the lipid profiles. ${ }^{14)}$ As a plausible mechanism, prolonged sedentary behavior suppresses the activities of lipoprotein lipase-a protein important for controlling plasma triglyceride catabolism, HDL cholesterol, and other metabolic risk factors. Low lipoprotein lipase is associated with blunted plasma triglyceride uptake and reduced plasma HDL cholesterol levels. ${ }^{15)}$

In contrast to our study, several studies reported significant associations between sedentary time and glucose- and insulin-related markers. ${ }^{5,8,16,17)}$ A study among Latino/Hispanic adults found that sedentary time is associated with fasting and 2-hour glucose, as well as fasting insulin and homeostatic model assessment of insulin resistance, after exclusion of individuals who were diagnosed with or taking medications for cardiovascular disease. ${ }^{13)}$ An animal study reported a decline in whole-body insulin sensitivity and an increase in visceral fat amount when physically active rats stop high levels of exercise. ${ }^{18)}$

No gold standard for sedentary behavior assessment has been established. ${ }^{19)}$ In this study, we measured sedentary time using a standardized self-administered questionnaire. Recent studies suggested that quantification of sedentary time and physical activity is necessary, such as by using an accelerometer. Another point is that patterns of sedentary time may have differential effects on cardiovascular risks. A study in a large representative sample of Canadian adults reported that breaking-up sedentary time is more related to cardio-metabolic variables than total sedentary time. ${ }^{16)}$ A recent study found that the association between sedentary time and cardio-metabolic risks becomes insignificant after adjustment for overall physical activity measured using an accelerometer, instead of MVPA. ${ }^{20)}$ Sedentary behavior usually refers to any waking behavior characterized by an energy expenditure $\leq 1.5 \mathrm{MET}^{2)}$ No currently used questionnaires assess physical activity levels between 1.5 METs (sedentary activity) and 3.0 METs (moderate physical activity). Thus, this study suggests that sedentary behavior may not have health effects independent of physical activity if overall physical activity is adjusted. In addition, types of sedentary activities may have different health effects. A recent large prospective study found that watching TV is associated with an increased cardiovascular mortality; however, time spent sitting in a car or bus and at work is not related to mortality. This finding proposes that sedentary behavior may consist of different natures. ${ }^{21)}$

The present study has several strengths. To the best of our knowledge, this study is the first to investigate relationships between sedentary time and cardiovascular risk factors in a large representative sample of Korean adults. We applied sample design variables and weights in analyses to represent the entire Korean population. We also adjusted for various sociodemographic and health-related variables that may affect the relationships between sedentary time and cardio-metabolic risk factors. ${ }^{22)}$ Compared with most of the previous studies that included individuals with preexisting cardiovascular disease and adjusted for the disease histories, we excluded those who were diagnosed with cardio-metabolic diseases or cancers to reduce reverse- 
causality between sedentary time and cardio-metabolic markers.

The potential limitations of this study are as follows. First, we used self-reported data on sedentary time and physical activity assessed using IPAQ, a validated and widespread questionnaire. ${ }^{23)}$ However, we cannot exclude the possibility that using self-report to quantify activity may underestimate the strength of relationships with risk factors. ${ }^{24)}$ Another issue is that no currently used questionnaires measure 1.5-3.0 METs of physical activities. Second, we did not evaluate the effects of breaking-up sedentary time. Lastly, this was a cross-sectional study; the design precluded casual inferences about the relationships.

In conclusion, prolonged sedentary time was significantly associated with high DBP and low HDL cholesterol level in Korean adults. The associations were independent of general and abdominal obesity and MVPA levels. Future longitudinal investigations on the association between sedentary lifestyle and cardiovascular outcomes in Korean population are warranted to confirm the present results.

\section{CONFLICT OF INTEREST}

No potential conflict of interest relevant to this article was reported.

\section{REFERENCES}

1. Hill JO, Wyatt HR, Reed GW, Peters JC. Obesity and the environment: where do we go from here? Science 2003;299:853-5.

2. Sedentary Behaviour Research Network. Letter to the editor: standardized use of the terms "sedentary" and "sedentary behaviours". Appl Physiol Nutr Metab 2012;37:540-2.

3. Ministry of Health and Welfare. Physical activity guidelines for Koreans. Sejong: Ministry of Health and Welfare; 2013.

4. Ford ES, Caspersen CJ. Sedentary behaviour and cardiovascular disease: a review of prospective studies. Int J Epidemiol 2012;41:1338-53.

5. Healy GN, Matthews CE, Dunstan DW, Winkler EA, Owen N. Sedentary time and cardio-metabolic biomarkers in US adults: NHANES 2003-06. Eur Heart J 2011;32:590-7.

6. Crichton GE, Alkerwi A. Physical activity, sedentary behavior time and lipid levels in the Observation of Cardiovascular Risk Factors in Luxembourg study. Lipids Health Dis 2015;14:87.

7. Salahuddin UI, Pandey A, Ayers CR, Kulinski J, Garg S, Kumbhani DJ, et al. Dose response relationship between sedentary time and cardiovascular disease risk: a meta-analysis. Circulation 2015;132:A17035.

8. Altenburg TM, Lakerveld J, Bot SD, Nijpels G, Chinapaw MJ. The prospective relationship between sedentary time and cardiometabolic health in adults at increased cardiometabolic risk: the Hoorn Prevention Study. Int J Behav Nutr Phys Act 2014;11:90.

9. Byun W, Dowda M, Pate RR. Associations between screen-based sedentary behavior and cardiovascular disease risk factors in Korean youth. J Korean Med Sci 2012;27:388-94.

10. International Physical Activity Questionnaire Group. Guidelines for data processing and analysis of the International Physical Activity Questionnaire (IPAQ): short and long forms [Internet]. [place unknown]: International Physical Activity Questionnaire Group; 2005 [cited 2015 Dec 12]. Available from: https://docs.google.com/viewer?$\mathrm{a}=\mathrm{v} \&$ pid=sites\&srcid=ZGVmYXVsdGRvbWFpbnx0aGVpcGFxfGd4Oj E0NDgxMDk3NDU1YWRIZTM.

11. National Institute on Alcohol Abuse and Alcoholism. National Institute on Alcohol Abuse and Alcoholism (NIAAA) guideline. Bethesda (MD): National Institute on Alcohol Abuse and Alcoholism; 2005.

12. Gopinath B, Baur LA, Hardy LL, Kifley A, Rose KA, Wong TY, et al. Relationship between a range of sedentary behaviours and blood pressure during early adolescence. J Hum Hypertens 2012;26:350-6.

13. Qi Q, Strizich G, Merchant G, Sotres-Alvarez D, Buelna C, Castaneda $\mathrm{SF}$, et al. Objectively measured sedentary time and cardiometabolic biomarkers in US Hispanic/Latino adults: the Hispanic Community Health Study/Study of Latinos (HCHS/SOL). Circulation 2015;132: 1560-9.

14. Brocklebank LA, Falconer CL, Page AS, Perry R, Cooper AR. Accelerometer-measured sedentary time and cardiometabolic biomarkers: a systematic review. Prev Med 2015;76:92-102.

15. Hamilton MT, Hamilton DG, Zderic TW. Role of low energy expenditure and sitting in obesity, metabolic syndrome, type 2 diabetes, and cardiovascular disease. Diabetes 2007;56:2655-67.

16. Carson V, Wong SL, Winkler E, Healy GN, Colley RC, Tremblay MS. Patterns of sedentary time and cardiometabolic risk among Canadian adults. Prev Med 2014;65:23-7.

17. Gennuso KP, Gangnon RE, Matthews CE, Thraen-Borowski KM, Colbert LH. Sedentary behavior, physical activity, and markers of health in older adults. Med Sci Sports Exerc 2013;45:1493-500.

18. Booth FW, Laye MJ, Lees SJ, Rector RS, Thyfault JP. Reduced physical activity and risk of chronic disease: the biology behind the consequences. Eur J Appl Physiol 2008;102:381-90.

19. Young DR, Hivert MF, Alhassan S, Camhi SM, Ferguson JF, Katzmarzyk PT, et al. Sedentary behavior and cardiovascular morbidity and mortality: a science advisory from the American Heart Association. Circulation 2016;134:e262-79.

20. Maher C, Olds T, Mire E, Katzmarzyk PT. Reconsidering the sedentary behaviour paradigm. PLoS One 2014;9:e86403.

21. Kim Y, Wilkens LR, Park SY, Goodman MT, Monroe KR, Kolonel LN. Association between various sedentary behaviours and all-cause, cardiovascular disease and cancer mortality: the Multiethnic Cohort Study. Int J Epidemiol 2013;42:1040-56.

22. Rhodes RE, Mark RS, Temmel CP. Adult sedentary behavior: a systematic review. Am J Prev Med 2012;42:e3-28.

23. Rosenberg DE, Bull FC, Marshall AL, Sallis JF, Bauman AE. Assessment of sedentary behavior with the International Physical Activity Questionnaire. J Phys Act Health 2008;5 Suppl 1:S30-44.

24. Celis-Morales CA, Perez-Bravo F, Ibanez L, Salas C, Bailey ME, Gill JM. Objective vs. self-reported physical activity and sedentary time: effects of measurement method on relationships with risk biomarkers. PLoS One 2012;7:e36345. 